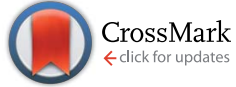

Cite this: RSC Adv., 2017, 7, 14078

Received 6th December 2016 Accepted 23rd February 2017

DOI: $10.1039 / c 6 r a 27846 a$

rsc.li/rsc-advances

\section{Physisorbed versus chemisorbed oxygen effect on thermoelectric properties of highly organized single walled carbon nanotube nanofilms $\uparrow$}

\author{
Milad Yarali, ${ }^{a} \mathrm{Ji} H a o,{ }^{\text {b }}$ Mohammad Khodadadi, ${ }^{a}$ Hatem Brahmi, ${ }^{\text {a }}$ Shuo Chen, ${ }^{\text {cd }}$ \\ Viktor G. Hadjiev, ${ }^{d}$ Yung Joon Jung ${ }^{b}$ and Anastassios Mavrokefalos*a
}

\begin{abstract}
The effect of physisorbed vs. chemisorbed oxygen on highly organized single walled carbon nanotube (SWCNT) ultrathin films is investigated by correlating the thermoelectric properties measured by a suspended micro-device to the SWCNT structure characterized by Raman spectroscopy and transmission electron microscopy. The results show that SWCNTs with weakly bonded oxygen molecules on the surface were determined to be initially $p$-type with metallic behavior and after annealing in vacuum they transition to $n$-type with semiconducting behavior where the charge transport is dominated by a 2D Mott variable range hopping mechanism due to molecular desorption. The structural characterization reveals that there is no change in the structure of the SWCNT network, indicating that the source of the drastic change in electrical properties is due to the molecule interaction with the surface of the SWCNT. Even though there is a significant change in the electrical properties, the thermal conductivity remains unchanged. On the other hand, the oxidized SWCNT sample with stronger $\mathrm{C}-\mathrm{O}$ bonds exhibits purely $\mathrm{p}$-type metallic behavior that is insensitive to annealing conditions and shows lower thermal conductivity values because of the enhanced phonon scattering due to the absorbed oxygen molecules and residual poly-methyl-methacrylate (PMMA).
\end{abstract}

\section{Introduction}

The importance of single walled carbon nanotube (SWCNT) networks in electronic and thermal applications cannot be understated. Recently, designing engineered carbon nanotube architectures composed of aligned nanotubes (horizontal or vertical) has attracted considerable interest due to broad applications in flexible electronic, ${ }^{1,2}$ optoelectronic, ${ }^{3}$ field emission ${ }^{4}$ and energy storage devices. ${ }^{5}$ The performance of these architectures is highly dependent on the exact structure of the individual nanotubes, their interactions within the network as well as their chemical interactions with the surrounding environment. ${ }^{6}$ To more efficiently design the next generation of micro-macroscopic functional devices, clarifying the thermoelectric properties such as thermal conductivity $(\kappa)$, electrical conductivity $(\sigma)$, and Seebeck coefficient $(S)$ of these aligned CNT network is crucial.

${ }^{a}$ Department of Mechanical Engineering, University of Houston, Houston, TX, 77204, USA. E-mail: amavrokefalos@uh.edu; Tel: +17137434531

${ }^{b}$ Department of Mechanical and Industrial Engineering, Northeastern University, Boston, MA, 02115, USA

${ }^{c}$ Department of Physics, University of Houston, Houston, TX, 77204, USA

${ }^{d}$ Texas Center for Superconductivity at the University of Houston (TCSUH), Houston, TX 77204, USA

$\uparrow$ Electronic supplementary information (ESI) available. See DOI: 10.1039/c6ra27846a
Over the last two decades, a great number of investigations have been conducted in studying various thermal and electrical properties of individual single-walled carbon nanotubes, ${ }^{7-16}$ multiwalled carbon nanotubes (MWCNTs), ${ }^{17-19}$ nanotube composites, ${ }^{20-24}$ and films..$^{25-32}$ These studies are essential in quantifying the various external or intrinsic effects on the individual phonon or charge transport properties of these seminal materials. To understand their interdependence, on the other hand, it is essential to perform multiple property measurements on the same samples. Specifically by performing simultaneous measurement of thermoelectric properties such as thermal conductivity, electrical conductivity and Seebeck coefficient on the same sample, allows us to quantify both the phonon and electron transport properties as well as their interdependence not otherwise possible by single property measurements. ${ }^{33,34}$ Even though these types of measurements were performed on individual single- and multiwalled carbon nanotubes ${ }^{35,36}$ and supported bulk films with broader size distribution of randomly oriented and aligned arrays of SWCNTs and MWCNTs; ${ }^{37-41}$ measurements of thin and highly organized suspended films composed of SWCNT bundles, that can have a significant impact in a broad range of technologies as mentioned earlier are notably absent.

These thermoelectric properties of SWCNTs have shown to be extremely sensitive to their surrounding molecular environment, specifically oxygen, which can have detrimental effect to their intrinsic properties. ${ }^{31,32}$ Unfortunately, there are 
contradictory reports on the exact effect of oxygen adsorption on the SWCNT properties. Some studies which were performed on the bulk films composed of randomly oriented SWCNTs showed that adsorbed oxygen molecules on the tubes surface dope the nanotubes positively due to charge transfer between carbon atoms and oxygen..$^{31,32,40-42}$ They claimed that oxygen removal by high temperature annealing of SWCNTs under ultrahigh vacuum or inert gas likely leads to hole dedoping from SWCNT. In contrast to this view, it has also been proposed that oxygen does not induce hole doping in SWCNTs..$^{43}$ Derycke et al. ${ }^{44,45}$ and Heinze et al. ${ }^{46}$ have argued that p-type to n-type transition in electronic character of SWCNT field effect transistor (CNTFET) by annealing is because of the barrier change at the metal-semiconductor contacts due to oxygen desorption. Therefore, further work is required to determine which process leads to the observed changes in the transport properties of SWCNTs.

Moreover, although most of the previous works just focus on the effect of physisorbed oxygen on electrical conductivity and Seebeck coefficient of thick (macroscopic) SWCNT mats or films, its effect to thermal transport is notably absent. To the best of our knowledge there are only two contradicting reports on the effect of oxygen adsorption of multi-walled CNTs (MWCNTs) $)^{39,47}$ and none investigating its effect on SWCNTs where we are reporting for the first time. Jin et al. ${ }^{39}$ showed an increase in the thermal conductivity with the desorption of oxygen for MWCNTs that they attributed to the decrease in the resistivity of the thermal junctions between the tubes, whereas Brown et al. ${ }^{47}$ showed an increase in the thermal conductance of MWCNTs with oxygen adsorption which they attributed to the deformation of $\mathrm{C}-\mathrm{C}$ bonds that affects the phonon modes of nanotubes.

In this work, we investigate the effect of physisorbed $v s$. chemisorbed oxygen on thermoelectric properties of ultrathin films composed of highly organized SWCNT bundles by utilizing a suspended microdevice. This method allows us to measure the thermal conductivity, electrical conductivity and Seebeck coefficient based on a four-probe thermoelectric measurement procedure reported in details elsewhere that accounts for the thermal contact resistance between the sample and the device. ${ }^{48}$ Therefore, the properties presented here are the intrinsic sample properties without the uncertainties of electrical or thermal contact resistances. Through correlating the thermoelectric properties to the SWCNT structure characterized by Raman spectroscopy and Transmission Electron Microscopy (TEM) and by fitting the measured thermoelectric properties with a two-band model, we are able to quantify how the physisorbed $v s$. chemisorbed oxygen molecules adsorbed to the CNT surface affect the thermoelectric properties. The results show that desorption of the weakly bonded oxygen molecules on the surface of SWCNTs after vacuum annealing changes the electrical behavior from metallic p-type to semiconducting n-type while its thermal conductivity remains unchanged, contradictory with reports on MWCNTs. On the contrary, purely p-type metallic behavior and lower thermal conductivity values were observed for oxidized SWCNT network.

\section{Experimental}

\subsection{Sample preparation}

In order to design and build horizontally organized SWCNT network on $\mathrm{SiO}_{2} / \mathrm{Si}$ substrates, a template-guided fluidic assembly method was employed. First, a plasma treatment was used to enhance the hydrophilic nature of $\mathrm{Si}$ or $\mathrm{SiO}_{2}$ surface. Then a photoresist film, providing hydrophobic surface on the Si or $\mathrm{SiO}_{2}$ substrate was patterned using optical or electronbeam lithography techniques and vertically submerged into the SWNT solution using a dip-coater and then gradually lifted from the solution with a constant pulling speed. We used the SWCNT-DI (de-ionized) water dispersion (concentration of 0.23 $w t \%)$ that was purchased from Brewer Science Inc. (CNTRENETM C100). The nanotubes were CVD grown and of mixed chirality in nature. The typical length of the SWCNTs was specified to be between $0.8 \mu \mathrm{m}$ and $1 \mu \mathrm{m}$, with an average diameter of about $1 \mathrm{~nm}$. Our own AFM and Raman spectroscopy measurements were mostly in agreement with about $\sim 10 \%$ deviation in specified length range and diameter. According to the vendor, the SWCNTs were not purified, and the dispersion was a mixture containing a natural ratio of metallic and semiconducting nanotubes, which can be assumed to be approximately $2 / 3$ rd semiconducting and $1 / 3$ rd metallic in nature. These SWCNTs were terminated with carboxylic acid groups, which adsorb ions such as $\mathrm{H}^{+}$and $\mathrm{OH}^{-}$from the aqueous solution leading to the presence of a net charge on the surface of SWCNTs. Using such a lithographically patterned template assisted dip-coating method, SWCNTs were directly assembled on hydrophilic surface, between pre-designed photoresist channels, forming organized SWCNT lateral networks in diverse geometries with feature sizes ranging from $100 \mathrm{~nm}$ to few microns. ${ }^{49}$

\subsection{Device fabrication}

The suspended microdevice used to perform the in situ measurements is illustrated in Fig. 1(a). The device incorporates two adjacent silicon nitride $\left(\operatorname{SiN}_{x}\right)$ membranes suspended by six $\operatorname{SiN}_{x}$ beams over a through-substrate hole. Two platinum (Pt) electrodes are patterned on each membrane to measure the electrical conductivity in a four-probe configuration and eliminate the contact electrical resistance. A serpentine Pt resistance thermometer is patterned on each membrane for measuring the thermal conductivity and Seebeck coefficient based on a fourprobe thermoelectric measurement procedure. The throughsubstrate hole under the two membranes allowed us to conduct TEM measurement of the SWCNT network assembled on the suspended device.

\subsection{Measurement procedure}

The widely used poly(methyl methacrylate) (PMMA)-assisted transfer technique ${ }^{50}$ was used to transfer horizontally organized SWCNTs onto a suspended measurement microdevice. To enhance the adhesion between samples and the membranes, a defined area of PMMA located on each membrane was polymerized, as shown in the Fig. 1(b). This was done by exposing 


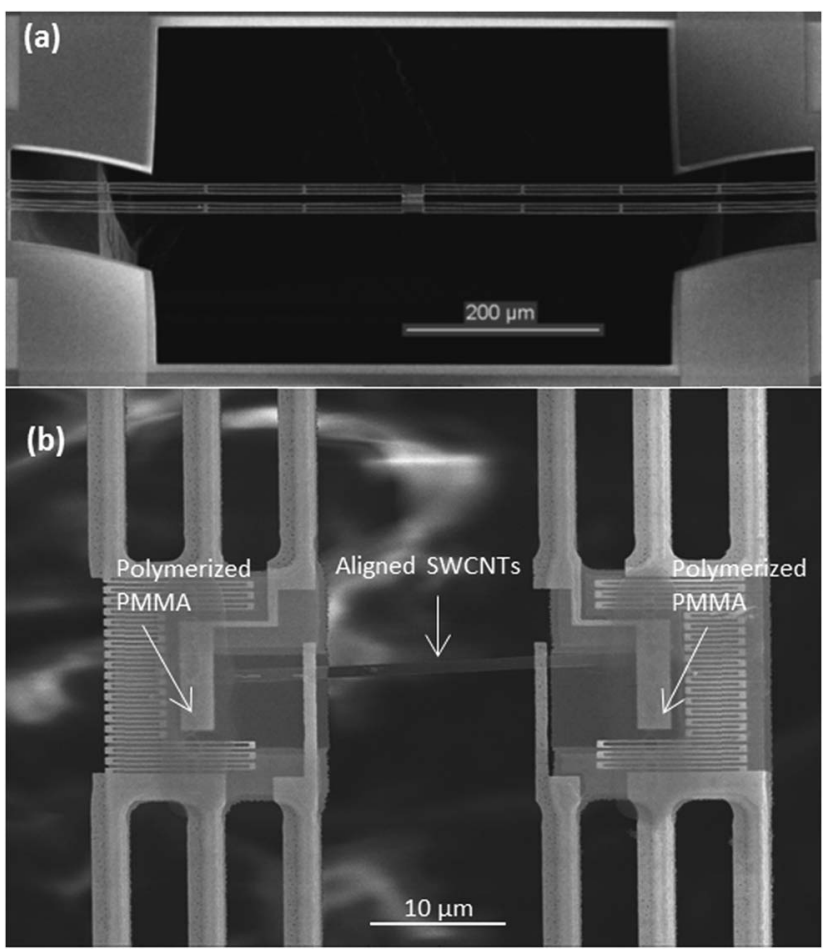

Fig. 1 (a) SEM image of the suspended microdevice, (b) SEM close-up of aligned SWCNT network assembled between the membranes. PMMA polymerization area is pointed on each membrane.

a small concentrated area of the PMMA to $15 \mathrm{kV}$ electron beam for 1 minute in a scanning electron microscope (SEM). This polymerization allowed us to anchor the samples on the membranes and guaranteed their attachment during PMMA removal by hot acetone.

A total of two highly organized SWCNT nanofilms are reported in this work, denoted as sample 1 and sample 2. The thermal conductance and Seebeck coefficient measurements were conducted on the samples in a vacuum-pumped cryostat according to the previously described procedure. ${ }^{33,48,51-53}$ Both samples were in contact with all four Pt electrodes on the two suspended membranes. The four-probe electrical resistance of the samples was measured by using the two outer Pt electrodes as current leads and the two inner Pt electrodes to measure the voltage drop.

Sample 1 was previously air saturated under ambient conditions for several days and then the measurement was conducted for a temperature range of 300-425 $\mathrm{K}$ ascending in temperature every $25 \mathrm{~K}$ (heating path). We then kept the sample at $425 \mathrm{~K}$ in vacuum $\left(10^{-7} \mathrm{mbar}\right)$ overnight and performed the measurements again starting from $400 \mathrm{~K}$ to $300 \mathrm{~K}$ descending in temperature every $25 \mathrm{~K}$ (cooling path). Sample 2 was annealed in air at $350{ }^{\circ} \mathrm{C}$ for 3 hours before conducting the measurements from 300-425 $\mathrm{K}$. As discussed later we also annealed the sample in vacuum $\left(10^{-7} \mathrm{mbar}\right)$ overnight to try and reproduce similar results as in sample 1. Both samples were kept at each temperature for 3 to 4 hours to reach steady state condition and then 3 sets of Seebeck coefficient data, each of which took approximately 15 minutes, were taken for each temperature consecutively.
Table 1 Dimensions of the suspended SWCNT films measured in this work

\begin{tabular}{llll}
\hline Sample & $\begin{array}{l}\text { Suspended length } \\
(\mu \mathrm{m})\end{array}$ & $\begin{array}{l}\text { Width } \\
(\mu \mathrm{m})\end{array}$ & $\begin{array}{l}\text { Thickness } \\
(\mathrm{nm})\end{array}$ \\
\hline 1 & $15.21 \pm 0.08$ & $1.05 \pm 0.099$ & $19 \pm 2.48$ \\
2 & $7.97 \pm 0.052$ & $0.95 \pm 0.074$ & $19 \pm 2.48$
\end{tabular}

Upon completion of thermoelectric measurements, the samples were removed from the cryostat and then TEM and Raman spectroscopy were performed to correlate the properties to structure. Dimensions of the suspended segment of the samples were measured using SEM and atomic force microscopy (AFM) (see Fig. S1 in the ESI $\dagger$ ) and tabulated in Table 1. These dimensions were used to calculate the thermal conductivity and electrical conductivity from the measured thermal conductance and electrical resistance.

\section{Results and discussion}

\subsection{Structural characterization}

TEM and SEM images of sample 1 are shown in Fig. 2(a). No significant PMMA residues were observed on the film and the structure of this sample remains unchanged upon PMMA transferring and thermoelectric characterization. This is also verified by Raman spectroscopy characterization shown in Fig. S3 (see ESI $\dagger$ for more details). Sample 2 on the other hand shows some sample deformation as well as the presence of PMMA residues on the surface as seen in Fig. 2(b). Unfortunately thermal annealing in air was found to be ineffective to burn out these residues. Similar polymer residue was observed on the surface of suspended graphene or h-BN samples that were in contact with a PMMA layer during sample preparation. ${ }^{54-56}$ The effect of this unwanted polymer residues on the thermoelectric properties of this sample will be discussed later.

To fully characterize the sample structures beyond TEM, we carried out Raman spectroscopy characterization of both samples after thermoelectric characterization. The Raman spectra allow us to obtain the SWCNTs diameter distribution, ${ }^{57}$ electronic type (semiconducting or metallic), ${ }^{58}$ appearance of oxidation induced defects, ${ }^{59}$ and the degree of nanotube alignment along the longest side of a ribbon. Fig. 3(a) shows the Raman spectra of radial breathing mode (RBM) in the nanofilms excited with three different laser lines. The RBM is a characteristic phonon mode of a nanotube in which all atoms vibrate in radial direction. The RBM phonon frequency $\omega_{\mathrm{RBM}} \propto$ $1 / d$ and the resonant optical transition energies $E_{\mathrm{ii}}$ determines uniquely the nanotube diameter $d$ and its electronic type. ${ }^{57,58}$ The RBM of semiconducting and metallic nanotubes in Fig. 3(a) are denoted by $S_{\mathrm{ii}}$ and $M_{\mathrm{ii}}$, respectively, when the laser energy is close to $E_{\mathrm{ii}}$. For the given diameter distribution in sample 1, the laser line at $514 \mathrm{~nm}$ excites predominantly semiconducting SWCNTs whereas that at $633 \mathrm{~nm}$ mostly the metallic ones. The weaker RBM spectra of sample 2 excited with $633 \mathrm{~nm}$ line is due to smearing of the resonances by increased number of defects. The latter is also confirmed by the D- and G-mode Raman 

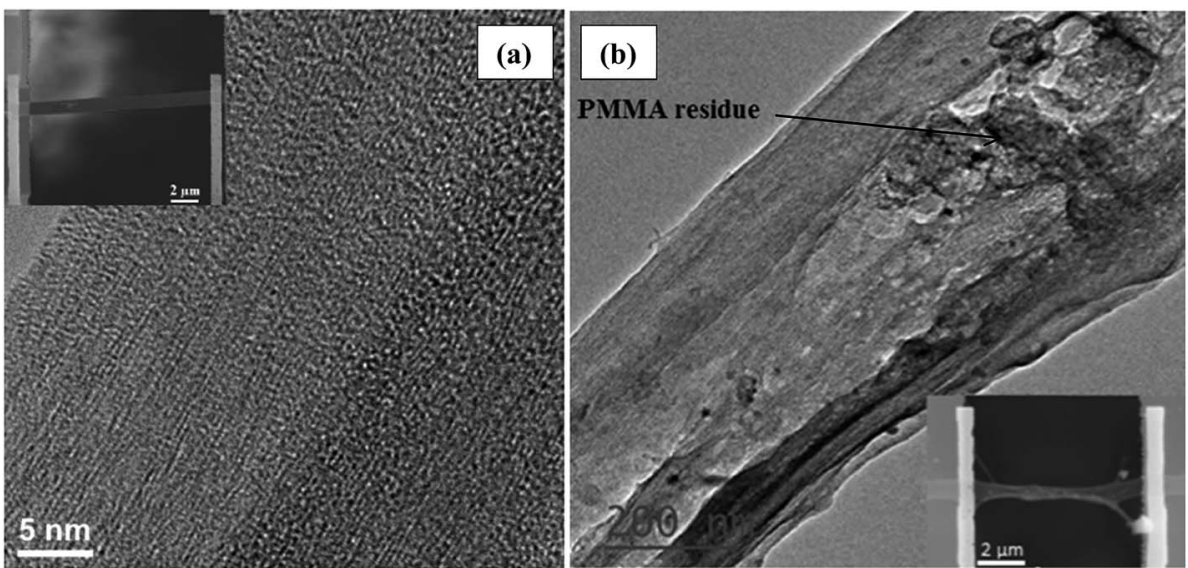

Fig. 2 TEM and SEM images of (a) samples 1 and (b) sample 2. The PMMA residue is pointed out by arrow.

spectra shown in Fig. 3(b). The spectra of metallic nanotubes excited with $633 \mathrm{~nm}$ exhibit strong increase of the "defect" D-band. On the other hand, the oxidation of the semiconducting nanotubes, seen under $514 \mathrm{~nm}$ excitation, results in their protonation, p-type doping, with associated nanotube shrinkage and the corresponding increase of G-mode frequency..$^{59}$

The alignment of SWCNTs along the longest side $R$ of nanofilm ribbons was quantitatively determined by measuring the variation of G-mode Raman intensity $I_{\mathrm{VV}}$ of SWNTs with angle $\Psi$ between the $R$ direction and the polarization direction of incident light as shown in Fig. 4 . The inset in Fig. 4 displays a schematic diagram of the Raman configuration for measurement of angular dependence of the G mode intensity in back scattering configuration and for parallel incident, $e_{\mathrm{I}}$, and scattered, $e_{\mathrm{S}}$, light polarizations. In this experiment, the sample was rotated at angle $\Psi$ while keeping the parallel incident and scattered light polarization directions fixed. The deviation angle of SWCNTs with respect to $R$ is denoted by $\theta$. The line through the data for the $I_{\mathrm{Vv}}(\Psi) / I_{\mathrm{VV}}(0)$ plot presents a fit to the function $I_{\mathrm{VV}}=A+B \cos ^{2} \Psi+C \cos ^{4} \Psi$. The correspondence between fitting parameters $A, B$, and $C$ and orientation order parameters $P_{2}(\cos \theta)$ and $P_{4}(\cos \theta)$ is given by the expression: ${ }^{60}$
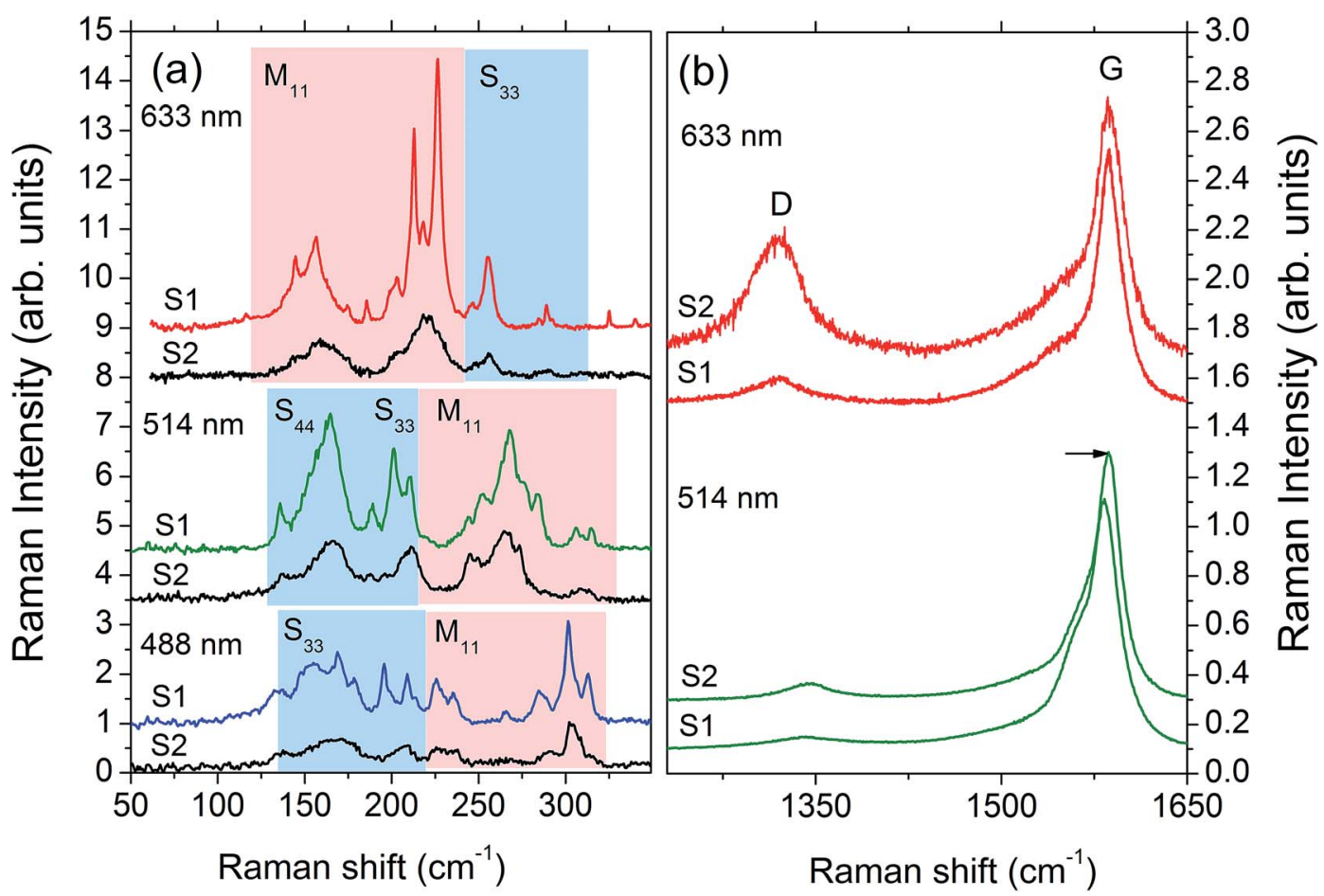

Fig. 3 (a) Raman spectra of radial breathing modes in samples 1 (S1) and 2 (S2) excited with three different laser lines. Frequency ranges of metallic (M11) and semiconducting (S33 and S44) SWCNT are shaded in pink and blue, respectively. (b) Raman spectra of D and G modes in S1 and S2 (oxidized SWCNT). Note the upward shift of the G mode in S2 when excited with $514 \mathrm{~nm}$ laser. 


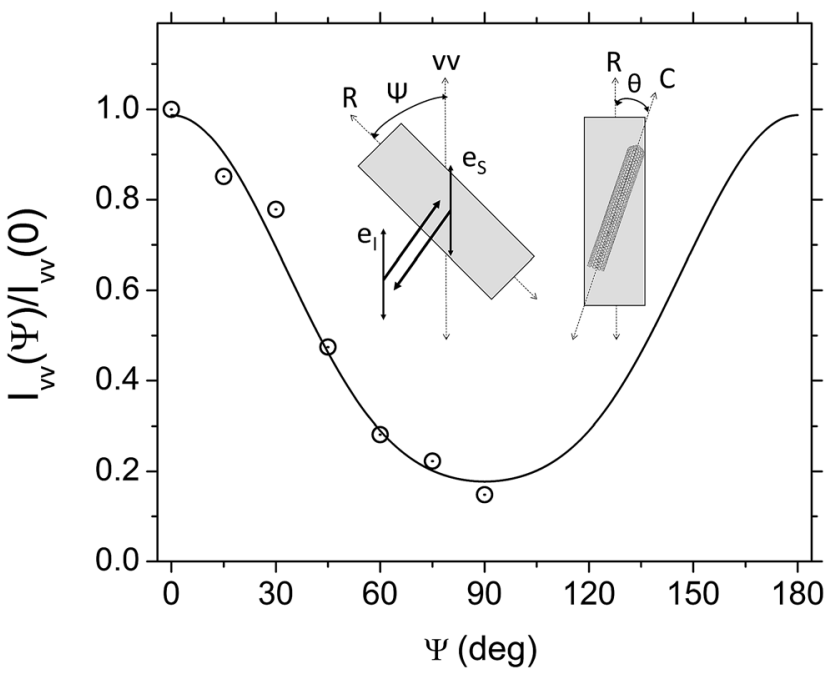

Fig. 4 Angular dependence of G mode intensity /Vv in sample 1. Intensities are obtained by deconvolution of the baseline corrected spectra using mixed Gaussian and Lorentzian functions. The line through the data for the $I_{V V}$ Vs. $\Psi$ plot corresponds to a fit to the function $I_{\mathrm{VV}}=A+B \cos ^{2} \Psi+C \cos ^{4} \Psi$. Schematic diagram: $e_{\mathrm{I}}$ and $e_{\mathrm{S}}$ are the direction of incident and scattered light polarizations, $R-$ direction of longest side of sample 1 ribbon, $\theta$ - angular deviation of SWCNTs from $R$.

$$
\begin{aligned}
I_{\mathrm{VV}}(\Psi) \propto\left[\left(\frac{6}{7}\right) \cos ^{2} \Psi-\frac{2}{7}\right]\left\langle P_{2}(\cos \theta)\right\rangle \\
\quad+\left[\cos ^{4} \Psi-\left(\frac{6}{7}\right) \cos ^{2} \Psi+\frac{3}{35}\right]\left\langle P_{4}(\cos \theta)\right\rangle+\frac{1}{5}
\end{aligned}
$$

$P_{2}(\cos \theta)$ and $P_{4}(\cos \theta)$ are the average values of second- and fourth-order Legendre polynomials, respectively, over $\theta$ from 0 to $\pi$. The orientation order parameter $P_{2}(\cos \theta)$ (also known as the Herman's orientation function) is a measure of the mean orientation angle, whereas $P_{4}(\cos \theta)$ is needed for a reconstruction of the orientation distribution function. SWCNTs in the sample 1 are relatively well aligned along $R$ having $P_{2}(\cos \theta)=$ 0.87 and $P_{4}(\cos \theta)=0.48$, perfectly aligned SWCNTs are characterized by $P_{2}(\cos \theta)=1$, whereas for randomly oriented ones $P_{2}(\cos \theta)=0$.

\subsection{Thermoelectric properties}

3.2.1. Electrical conductivity. Fig. 5 shows the variation of electrical conductivity versus temperature for both samples. As illustrated in Fig. 5(a) the electrical conductivity of sample 1 before annealing (heating path) shows inverse relation with the temperature from $300 \mathrm{~K}$ to $400 \mathrm{~K}$ and possess metallic behavior due to unintentional hole doping from the physisorbed oxygen. The measured electrical conductivity of sample 1 is comparable to reported values for aligned SWCNT films that fall in the range 6-128 S mm $\mathrm{mm}^{-1}$ depending on the chirality and extent of intrinsic defects. ${ }^{61-64}$ Deoxygenating by vacuum annealing localizes the charge carriers on the nanotubes indicating the presence of strong disorder at contact points between the bundles in the SWCNT network. ${ }^{65-67}$ In result, semiconducting behavior in electrical conductivity is observed and its transport mechanism can be fitted by Mott variable range hopping (VRH) mechanism. ${ }^{68}$ Moreover, because no significant structural change and PMMA residue were observed for sample 1 according to Fig. 2(a), the source of the drastic change in electrical properties is due to molecule desorption from the surface of the SWCNT.

Mott VRH model describes a system which is strongly disordered with the charge carriers hopping between localized states. $^{69}$ The VRH relationship between conductance and temperature is given by: ${ }^{68}$

$$
\sigma=\sigma_{0} \exp \left[-\left(\frac{T_{0}}{T}\right)^{\frac{1}{d+1}}\right]
$$

where $\sigma$ is the electrical conductivity, $\sigma_{0}$ is the prefactor that is usually considered to be weakly dependent on temperature, $d=$ 1,2 , or 3 is the dimensionality of the hopping conduction and $T_{0}$ is the characteristic temperature that is proportional to the energy separation between the available states. The upper temperature limit in which the Mott VRH can operate is determined by half of the Debye temperature $\left(T \leq \theta_{\mathrm{D}} / 2\right) \cdot{ }^{70}$ The Debye temperature of carbon nanotubes were reported to be $960 \mathrm{~K}$ and $1000 \mathrm{~K}^{71,72}$ Nonetheless, it is not entirely apparent what the correct Debye temperature value is for carbon nanotubes and it is expected to be likely identical to that of graphene, graphite and diamond..$^{73-76}$ Temperature dependent Debye temperature of graphite was studied by first-principle approaches and determined to be approximately $1600 \mathrm{~K}$ to $1700 \mathrm{~K}$ at our studied temperature range. ${ }^{77}$ Therefore, the Mott VRH can be applied in our case study and as shown in Fig. 5(b) the temperature dependent electrical conductivity of sample 1-cooling path is reproduced by VRH model with $d=2$ and $T_{0}=1351.8 \mathrm{~K}$.

Metallic behavior in electrical conductivity as depicted in Fig. 5(c) was observed for sample 2 because of oxidization by annealing in air environment. Also, sample 2 possesses smaller electrical conductivity when compared to sample 1 due to disruption of the $\pi$-bonded conduction path at either preexisting or newly created defect sites on the tubes during annealing process ${ }^{78,79}$ and the observed PMMA residue on the oxidized sample that affects the carrier mobility as it was well studied for graphene field-effect transistors (GFETs) by Suk et al. ${ }^{80}$ They observed experimentally that higher PMMA residue on transferred graphene samples results lower carrier mobility and can hence attribute to reduce electrical conductivity significantly.

3.2.2. Seebeck coefficient. Fig. 6 shows the measured Seebeck coefficient as a function of temperature for both samples used in this work. As illustrated in Fig. 6(a), sample 1 exhibits positive $S$ during the heating path and negative $S$ during the cooling path that indicates p-type and n-type behaviors, respectively. In the heating path $S$ increases slightly from $300 \mathrm{~K}$ to $350 \mathrm{~K}$ then starts decreasing significantly. After annealing at 425 K overnight, $S$ changes sign and slowly decreases with temperature reduction. This sign change of $S$ after high temperature annealing of SWCNTs under ultrahigh vacuum or inert gas was reported previously. ${ }^{31,32,40,41}$ In this study four probe configuration was used to eliminate the effect of barriers 

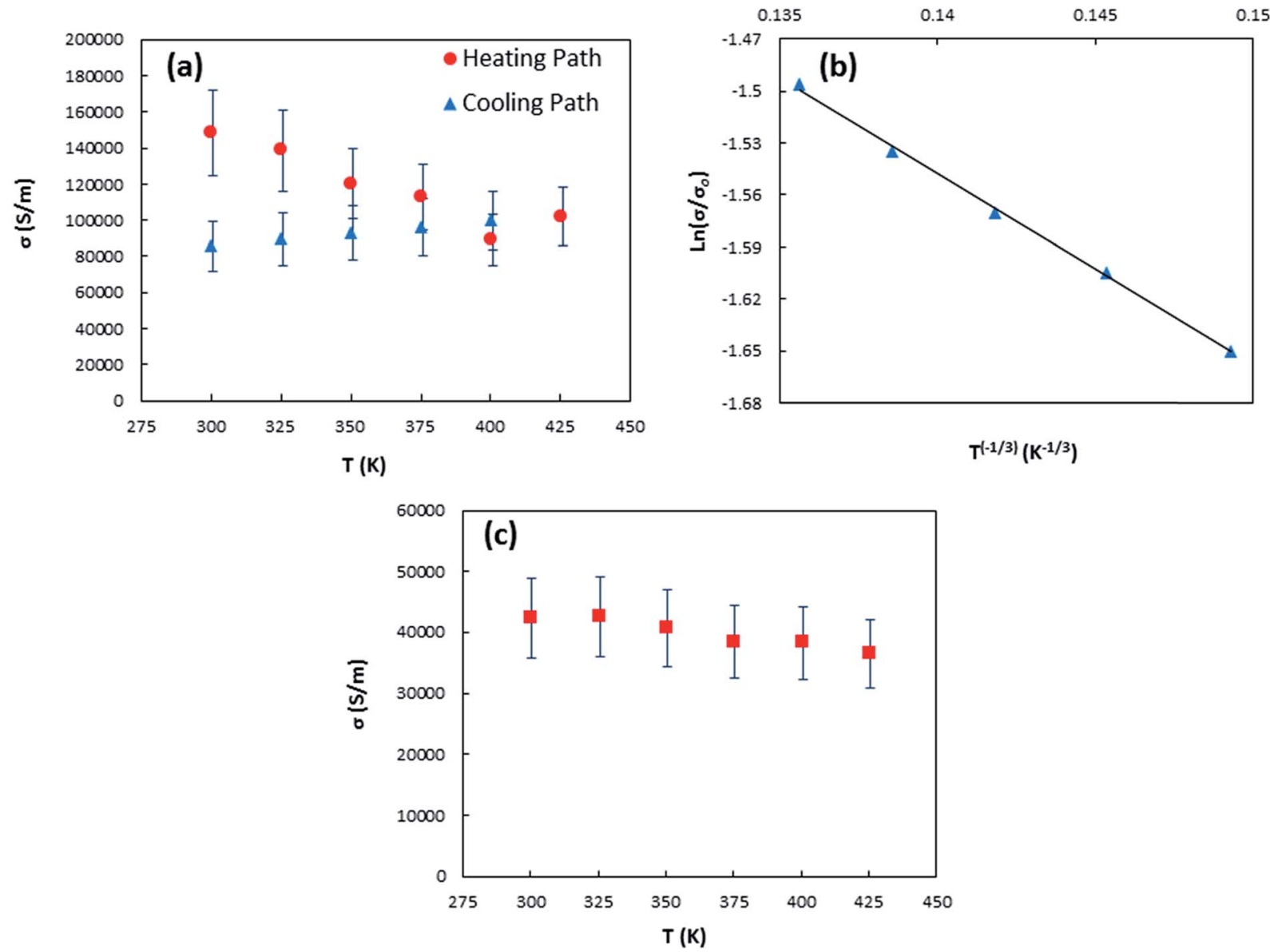

Fig. 5 (a) Temperature dependent electrical conductivity of sample 1 for both heating and cooling paths (b) fitting of sample 1-cooling path electrical conductivity with eqn (1) (c) temperature dependent electrical conductivity of sample 2.

at the metal-semiconductor contacts. Such a p- to n-type transition can hence be attributed to hole de-doping of SWCNT network by desorption of the physisorbed oxygen molecules in a high vacuum environment at elevated temperature (400 K). The details could be better explained by a change in density of states (DOS) at the Fermi level because of the charge transfer between the SWNTs and absorbed molecules. Unintentional doping of as-prepared SWCNT network from the ambient moves the Fermi level of the semiconducting tubes (S-SWCNT) inside or close to the valence band depending on doping level.
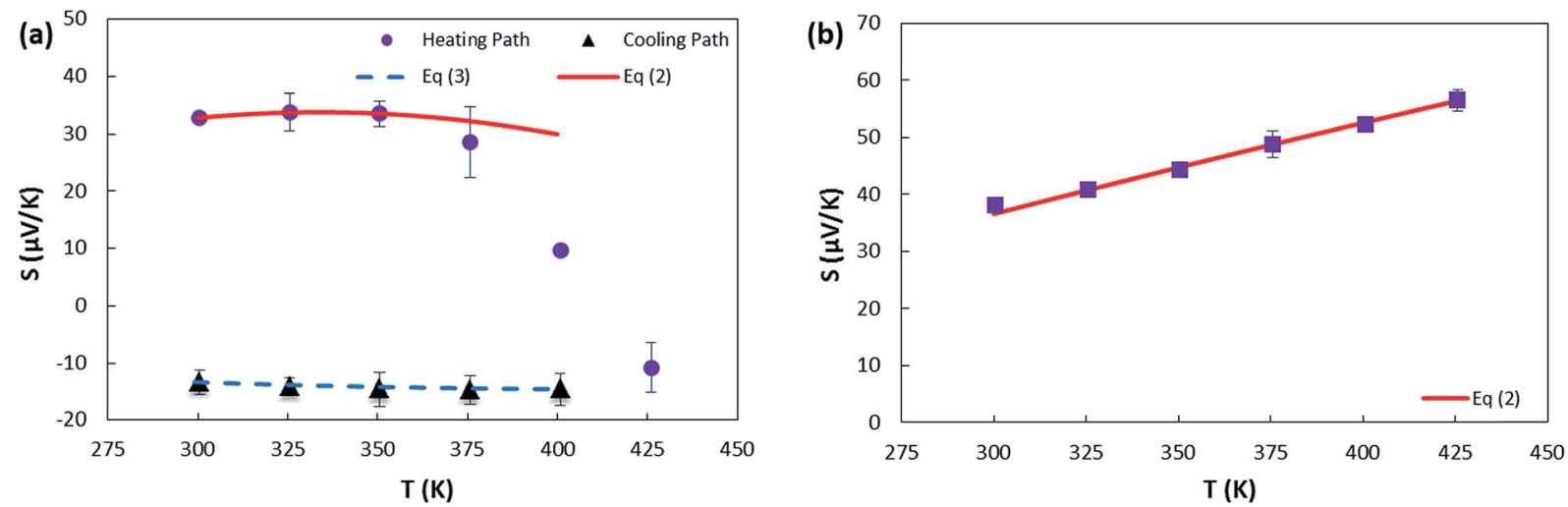

Fig. 6 The variation of Seebeck coefficient versus temperature for (a) sample 1. The solid and dashed lines represent fits to the data using eqn (2) and (3), respectively. The fitting parameters extracted from the fit of heating path to eqn (2) are $A=0.030 \mu \mathrm{V} \mathrm{K}-2 ; B=0.77 \mu \mathrm{V} \mathrm{K} \mathrm{K}^{-2} ; C=-0.95 \mu \mathrm{V}$ $\mathrm{K}^{-2} ; \lambda=600 \mathrm{~K}$. The fitting parameters extracted from the fit of cooling path to eqn (3) are $A=0.16 \mu \mathrm{V} \mathrm{K}-2 ; B=-47.46 \mu \mathrm{V} \mathrm{K}^{-(4 / 3)} ; T_{0}=1351.8 \mathrm{~K}$; $d=2$ (the values of $T_{0}$ and $d$ are determined from electrical conductivity data) (b) sample 2 . The fitting parameters extracted from the fit of heating path to eqn (2) are $A=0.04 \mu \mathrm{VK}^{-2} ; B=0.19 \mu \mathrm{VK}^{-2} ; C=0.06 \mu \mathrm{VK}^{-2} ; \lambda=400 \mathrm{~K}$. 
Vacuum annealing shifts the Fermi level towards intrinsic Fermi level by removing oxygen and moisture from S-SWCNTs. ${ }^{65}$ To verify the consistency of the sign change of the Seebeck coefficient, we reproduced this in another sample went through two heating/cooling cycles (see Fig. S4 in the ESI $\dagger$ for more details). Exposing the SWCNT network to ambient air after the first heating/cooling cycle leads to regain its p-type behavior (positive $S$ ) and again transition to n-type (negative $S$ ) by vacuum annealing.

As depicted in Fig. 6(b) sample 2 has large and positive Seebeck coefficient that increases with temperature as described in detail below. Annealing the sample overnight did not reproduce the sign change that occurred in sample 1 . According to Fig. 2(b), PMMA residues are observed on sample 2 that can cause weak p-doping as it was reported previously for graphene. $^{\mathbf{8 0 , 8 1}}$ However, these residues cannot prevent the transition from $\mathrm{p}$-type to $\mathrm{n}$-type upon high temperature vacuum annealing as it was found that a p-CNTFET protected by PMMA can be converted into an n-FET by annealing in a vacuum at $200{ }^{\circ} \mathrm{C}$ which is not stable under ambient air pressure. ${ }^{45}$ This implies that annealing the sample in air, the oxygen molecules are chemisorbed on the SWCNT surfaces and PMMA residues, oxidizing thus this sample, and results in a larger density of holes due to a greater number of strongly bound oxygen acceptors. This makes the SWCNT network permanently p-type so that even when vacuum annealing was applied, we were unable to change the $S$ sign.

3.2.3. Two band model. The temperature dependent $S$ can be used to better clarify the transport mechanism in the highly organized SWCNT nanofilm. The $S$ of a simple metal has a linear relation with $T$, while that of a semiconductor displays $1 / T$ dependence. $^{82}$ When variable range hopping is the conduction mechanism, $S$ can be described by $T^{(d-1) /(d+1)}$, where $d$ is the dimensionality of the system. ${ }^{83}$ The SWCNT network is inhomogeneous since it contains a random distribution of metallic and semiconducting elements. Romero et al. ${ }^{\mathbf{4 0}}$ experimentally observed that even though contact barrier between ropes in the SWCNT film can affect the total film resistance, it is not significantly involved in the $S$. Therefore; such an organized SWCNT network in this work can be modeled as comprising semiconducting and metallic tubes in parallel. According to a two-band model, the total $S$ can be given by the following equations depending on observed temperature dependent electrical conductivity. ${ }^{\mathbf{8 4 , 8 5}}$

$$
\begin{gathered}
S_{\text {tot }}=A T+(B \lambda+C T) \exp \left(-\frac{\lambda}{T}\right) \\
S_{\text {tot }}=A T+B T^{(d-1) /(d+1)} \exp \left[-\left(\frac{T_{0}}{T}\right)^{\frac{1}{d+1}}\right]
\end{gathered}
$$

where $A, B$ and $C$ are constant, $\lambda$ is the gap temperature measured from the midgap to the band edge, $d$ is dimensionality and $T_{0}$ is the characteristic temperature. In both equations the first term in the sum is the contribution of metallic tubes and the second is that of semiconducting tubes. Since the electrical conductivities of sample 1-heating path and sample 2 exhibit metallic behavior with $1 / T$ relation, the observed $S$ for these two cases were fitted with eqn (2). On the other hand, for sample 1 cooling path, as illustrated in Fig. 5(b) the electrical conductivity follows the VRH eqn (1) so eqn (3) was used to fit the measured $S$. The lines in Fig. 6 represent fits of eqn (2) and (3) and the fitting parameters are given in the caption of Fig. 6. Based on the calculated fitting parameters, it is noted that the metallic and semiconducting contributions for sample 1-heating path and sample 2 are hole-like which is the indicative of hole doping by ambient air. Also, the magnitudes $\lambda$ correspond to narrow energy gaps of 51.7 and $34.47 \mathrm{meV}$ for sample 1-heating path and sample 2, respectively. Higher carrier concentration in oxidized sample leads to smaller activation energy compared to that of sample $1 .^{86}$ Significantly the $B$ parameter in the fitting of eqn (3) to the sample 1-cooling path is negative demonstrating that the semiconducting contribution is electron-like and dominated due to hole de-doping.

3.2.4. Thermal conductivity. We further investigated the temperature dependence of thermal conductivity of both samples as shown in Fig. 7. Room temperature thermal conductivities of individual SWCNTs have been determined through experimental and molecule dynamic studies to be in the range of $2500-6600 \mathrm{~W} \mathrm{~m}^{-1} \mathrm{~K}^{-1} \cdot{ }^{7-9}$ Lower thermal conductivities in the range of $20-200 \mathrm{~W} \mathrm{~m}^{-1} \mathrm{~K}^{-1}$ was observed for bulk SWCNT films. ${ }^{37}$ Such a discrepancy can be attributed to various effects such as tube-tube contacts and increased phonon scattering due to defect density introduced during film preparation methods.

Our measured room temperature thermal conductivity of sample 1 is well matched with previously reported data in ref. 6 for the same highly organized SWCNT nanofilm and $>3$ times greater than the recently reported thermal conductivity of randomly oriented and nanometer thick supported ${ }^{\mathbf{8 7}}$ and suspended $^{88}$ SWCNT films. It is known that the thermal conductivity is dominated by phonons rather than electrons in the CNT networks throughout the whole temperature range. ${ }^{39,89,90}$ Since the maximum thermal conductivity occurs along the axis of CNT, fabricating CNT arrays in the aligned fashion could reduce the contact barriers and phonon scattering as a result. As shown in Fig. 7(a) the thermal conductivity of sample 1 remains unchanged after deoxygenating. This is contradictory with the report by Jin et $a .^{39}$ which has showed that for MWCNTs annealed in inert environment at $100{ }^{\circ} \mathrm{C}$ for 4 hours (comparable with our annealing conditions), a 3-fold enhancement in thermal conductivity at $300 \mathrm{~K}$ compared to the as grown samples, indicating that oxygen desorption increases thermal conductivity through reducing the resistivity of the thermal junctions between the tubes. It is also contradictory with the report by Brown et al. ${ }^{47}$ that showed physisorbed oxygen molecules enhance the thermal conductance of MWCNTs bundles by $20 \%$ due to deformation of $\mathrm{C}-\mathrm{C}$ bonds that affects the phonon modes of nanotubes as well as facilitating the inter-shell transfer of heat in the nanotubes. From our work, it can be concluded thus that the physisorbed oxygen molecules have negligible effect on the phonon transport in such ultrathin SWCNT film. However, for the chemisorbed sample we see a different trend, Fig. 7(b) depicts smaller thermal conductivity for sample 2 compared to that of sample 1 for the whole 
(a)

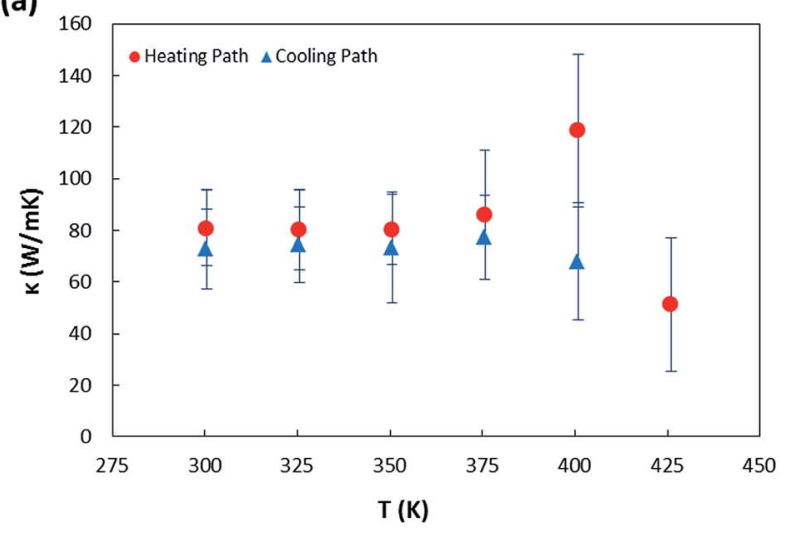

(b)

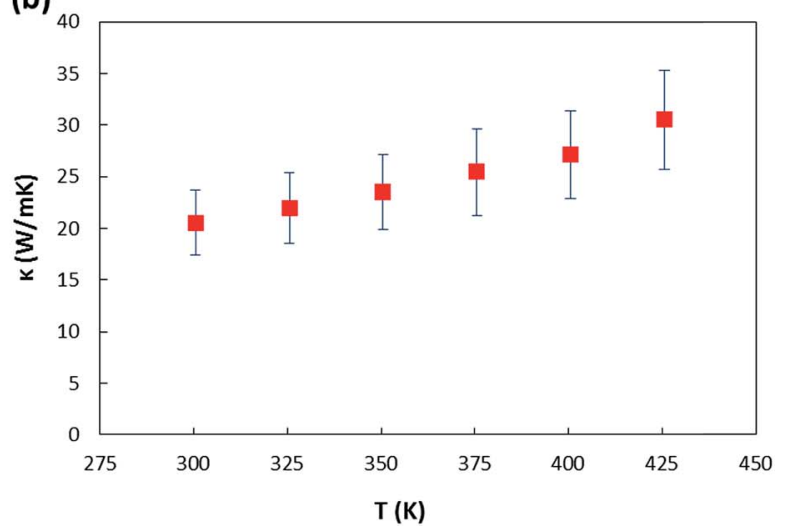

Fig. 7 (a) Thermal conductivity versus temperature for (a) sample 1 and (b) sample 2.

temperature range. The main factors responsible for providing phonon scattering sites and thereby the significant reduction in thermal conductivity of sample 2 are: 1 - chemisorbs oxygen molecules due to annealing in air that increase the $\mathrm{C}-\mathrm{O}$ bonds especially in the defect sites or open ends of CNTs and 2 - the presence of PMMA residue that was observed on suspended segment of this sample. The influence of latter one on scattering of phonons in suspended bilayer graphene was reported by Pettes et al. ${ }^{91}$ In addition, the thermal conductivity of sample 2 was observed to be monotonically increasing with the temperature in the range studied. It was investigated computationally that introduced defects on SWCNTs may affect the phonon dispersion relation and therefore the temperature at which the thermal conductivity peak occurs can be influenced. ${ }^{92}$ The mechanism of thermal conduction is more complicated for a SWCNT thin film with existence of impurities, as in the present sample.

\section{Conclusions}

This study presents in situ measurement of thermal and electrical conductivity and Seebeck coefficient of highly organized SWCNT nanofilms. Fluidic assembly method was employed to produce horizontally aligned SWCNT films with average thickness of $19 \mathrm{~nm}$. The results show that the SWCNT nanofilm with physisorbed oxygen molecules exhibits p-type with metallic behavior in electrical conductivity. Vacuum annealing leads to desorption of the oxygen molecule that transitions the nanofilms to n-type with semiconducting behavior in electrical conductivity that can be fitted with $2 \mathrm{D}$ variable range hopping. However, the thermal conductivity remains unchanged after the vacuum annealing. Since four probe configuration was utilized to perform the measurements and structural characterization revealed no change in the SWCNT film structure, we believe that this remarkable change in the thermoelectric properties of SWCNT nanofilm is purely due to oxygen desorption. Moreover, annealing the SWCNT films in air causes the oxygen molecule to chemisorb on the SWCNT nanofilm surface and (a) transforms the SWCNT nanofilm permanently p-type with metallic behavior in electrical conductivity and (b) significantly reduces the thermal conductivity by enhancing phonon scattering due to increase in the impurity concentration.

\section{Acknowledgements}

AM acknowledges financial support from University of Houston. Y. J. J. acknowledges the financial support from National Science Foundation-DMEREF (1434824) and Technology Innovation Program (10050481) funded by the Ministry of Trade, Industry \& Energy, Korea. V. G. H work was supported by the State of Texas through the Texas Center for Superconductivity at the University of Houston (TcSUH).

\section{Notes and references}

1 E. Artukovic, M. Kaempgen, D. S. Hecht, S. Roth and G. Grüner, Nano Lett., 2005, 5, 757-760.

2 J.-H. Ahn, H.-S. Kim, K. J. Lee, S. Jeon, S. J. Kang, Y. Sun, R. G. Nuzzo and J. A. Rogers, Science, 2006, 314, 1754-1757.

3 Z. Wu, Z. Chen, X. Du, J. M. Logan, J. Sippel, M. Nikolou, K. Kamaras, J. R. Reynolds, D. B. Tanner, A. F. Hebard and A. G. Rinzler, Science, 2004, 305, 1273-1276.

4 Y. J. Jung, S. Kar, S. Talapatra, C. Soldano, G. Viswanathan, X. Li, Z. Yao, F. S. Ou, A. Avadhanula, R. Vajtai, S. Curran, O. Nalamasu and P. M. Ajayan, Nano Lett., 2006, 6, 413-418. 5 R. Warren, F. Sammoura, K. S. Teh, A. Kozinda, X. Zang and L. Lin, Sens. Actuators, A, 2015, 231, 65-73.

6 H. Y. Jung, P. T. Araujo, Y. L. Kim, S. M. Jung, X. Jia, S. Hong, C. W. Ahn, J. Kong, M. S. Dresselhaus, S. Kar and Y. J. Jung, Nat. Commun., 2014, 5, 4941.

7 M. A. Osman and D. Srivastava, Nanotechnology, 2001, 12, 2124.

8 S. Berber, Y. K. Kwon and D. Tománek, Phys. Rev. Lett., 2000, 84, 4613-4616.

9 E. Pop, D. Mann, Q. Wang, K. Goodson and H. Dai, Nano Lett., 2006, 6(1), 96-100.

10 J. R. Lukes and H. Zhong, J. Heat Transfer, 2007, 129(6), 705716.

11 C. W. Padgett and D. W. Brenner, Nano Lett., 2004, 4(6), 1051-1053. 
12 N. Mingo and D. A. Broido, Nano Lett., 2005, 5(7), 1221-1225. 13 N. Mingo and D. A. Broido, Phys. Rev. Lett., 2005, 95, 096105. 14 R. Pan, Z. Xu, Z. Zhu and Z. Wang, Nanotechnology, 2007, 18(28), 285704.

15 C. Zhou, J. Kong and H. Dai, Phys. Rev. Lett., 2000, 84(24), 5604-5607.

16 A. Javey, M. Shim and H. Dai, Appl. Phys. Lett., 2002, 80(6), 1064-1066.

17 M. Fujii, X. Zhang, H. Xie, H. Ago, K. Takahashi, T. Ikuta, H. Abe and T. Shimizu, Phys. Rev. Lett., 2005, 95, 065502.

18 L. Lu, N. Kang, W. J. Kong, D. L. Zhang, Z. W. Pan and S. S. Xie, Phys. E, 2003, 18, 214-215.

19 N. Kang, L. Lu, W. J. Kong, J. S. Hu, W. Yi, Y. P. Wang, D. L. Zhang, Z. W. Pan and S. S. Xie, Phys. Rev. B: Condens. Matter Mater. Phys., 2003, 67, 033404-033408.

20 S. Shenogin, L. Xue, R. Ozisik, R. Keblinski and D. Cahill, J. Appl. Phys., 2004, 95, 8136-8144.

21 Q. Z. Xue, Nanotechnology, 2006, 17(6), 1655-1660.

22 S. U. S. Choi, Z. G. Zhang, W. Yu, F. E. Lockwood and E. A. Grulke, Appl. Phys. Lett., 2001, 79, 2252-2254.

23 M. J. Biercuk, M. C. Llaguno, M. Radosavljevic, J. K. Hyun, A. T. Johnsond and J. E. Fischer, Appl. Phys. Lett., 2002, 80, 2767-2769.

24 C. Guthy, F. Du, S. Brand, K. I. Winey and J. E. Fischer, J. Heat Transfer, 2007, 129(8), 1096-1099.

25 X. J. Hu, A. A. Padilla, J. Xu, T. S. Fisher and K. E. Goodson, J. Heat Transfer, 2006, 128(11), 1109-1113.

26 D. J. Yang, Q. Zhang, G. Chen, S. F. Yoon, J. Ahn, S. G. Wang, Q. Zhou, Q. Wang and J. Q. Li, Phys. Rev. B: Condens. Matter Mater. Phys., 2002, 66, 165440.

27 T. Tong, Y. Zhao, L. Delzeit, A. Kashani, M. Meyyappan and A. Majumdar, IEEE Trans. Compon. Packag. Technol., 2007, 30(1), 92-100.

28 M. L. Bauer, Q. N. Pham, C. B. Saltonstall and P. M. Norris, Appl. Phys. Lett., 2014, 105, 151909.

29 S. Shaikh, L. Li, K. Lafdi and J. Huie, Carbon, 2007, 45, 26082613.

30 M. A. Panzer, G. Zhang, D. Mann, X. Hu, E. Pop, H. Dai and K. E. Goodson, J. Heat Transfer, 2008, 130, 052401.

31 K. Bradley, S. H. Jhi, P. G. Collins, J. Hone, M. L. Cohen, S. G. Louie and A. Zettl, Phys. Rev. Lett., 2000, 85(20), 4361-4364.

32 P. G. Collins, K. Bradley, I. Masa and A. Zettl, Science, 2000, 287, 1801-1804.

33 A. Mavrokefalos, A. L. Moore, M. T. Pettes, L. Shi, W. Wang and X. Li, J. Appl. Phys., 2009, 105, 104318.

34 F. Zhou, J. Szczech, M. T. Pettes, A. L. Moore, S. Jin and L. Shi, Nano Lett., 2007, 7(6), 1649-1654.

35 C. Yu, L. Shi, Z. Yao, D. Li and A. Majumdar, Nano Lett., 2005, 5(9), 1842-1846.

36 P. Kim, L. Shi, A. Majumdar and P. L. McEuen, Phys. Rev. Lett., 2001, 87, 215502.

37 J. Hone, M. C. Llaguno, N. M. Nemes, A. T. Johnson, J. E. Fischer, D. A. Walters, M. J. Casavant, J. Schmidt and R. E. Smalley, Appl. Phys. Lett., 2000, 77, 666-668.

38 M. B. Jakubinek, M. A. White, G. Li, C. Jayasinghe, W. Cho, M. J. Schulz and V. Shanov, Carbon, 2010, 48, 3947-3952.
39 R. Jin, Z. X. Zhou, D. Mandrus, I. N. Ivanov, G. Eres, J. Y. Howe, A. A. Puretzky and D. B. Geohhegan, Phys. B, 2007, 388, 326-330.

40 H. E. Romero, G. U. Sumanasekera, G. D. Mahan and P. C. Eklund, Phys. Rev. B: Condens. Matter Mater. Phys, 2002, 65, 205410.

41 G. U. Sumanasekera, C. K. W. Adu, S. Fang and P. C. Eklund, Phys. Rev. Lett., 2000, 85(5), 1096-1099.

42 X. Li, J. S. Huang, S. Nejati, L. McMillon, S. Huang, C. O. Osuji, N. Hazari and A. D. Taylor, Nano Lett., 2014, 14, 6179-6184.

43 H. Ulbricht, G. Moos and T. Hertel, Phys. Rev. B: Condens. Matter Mater. Phys., 2002, 66, 075404.

44 V. Derycke, R. Martel, J. Appenzeller and P. H. Avouris, Appl. Phys. Lett., 2002, 80(15), 2773-2775.

45 V. Derycke, R. Martel, J. Appenzeller and P. H. Avouris, Nano Lett., 2001, 1, 453-456.

46 S. Heinze, J. Tersoff, R. Martel, V. Derycke, J. Appenzeller and P. H. Avouris, Phys. Rev. Lett., 2002, 89(10), 106801.

47 E. Brown, L. Hao, J. C. Gallop, J. C. Macfarlane, P. W. Josephs-Franks and L. F. Cohen, Phys. Status Solidi B, 2006, 243(13), 3380-3384.

48 A. Mavrokefalos, M. T. Pettes, F. Zhou and L. Shi, Rev. Sci. Instrum., 2007, 78, 034901.

49 B. Li, M. G. Hahm, Y. L. Kim, H. Y. Jung, S. Kar and Y. J. Jung, ACS Nano, 2011, 5(6), 4826-4834.

50 G. B. Barin, Y. Song, I. D. F. Gimenez, A. G. S. Filho, L. S. Barreto and J. Kong, Carbon, 2015, 84, 82-90.

51 L. Shi, D. Li, C. Yu, W. Jang, D. Kim, Z. Yao, P. Kim and A. Majumdar, J. Heat Transfer, 2003, 125, 881-888.

52 J. Zhou, C. Jin, J. H. Seol, X. Li and L. Shi, Appl. Phys. Lett., 2005, 87, 133109.

53 H. Brahmi, G. Katwal, M. Khodadadi, S. Chen, M. Paulose, O. K. Varghese and A. Mavrokefalos, Nanoscale, 2015, 7, 19004-19011.

54 I. Jo, M. T. Pettes, J. Kim, K. Watanabe, T. Taniguchi, Z. Yao and L. Shi, Nano Lett., 2013, 13, 550-554.

55 P. Y. Huang, C. S. Ruiz-Vargas, A. M. Van Der Zande, W. S. Whitney, M. P. Levendorf, J. W. Kevek, S. Garg, J. S. Alden, C. J. Hustedt, Y. Zhu, J. Park, P. L. McEuen and D. A. Muller, Nature, 2011, 469, 389-392.

56 Y. C. Lin, C. C. Lu, C. H. Yeh, C. H. Jin, K. Suenaga and P. W. Chiu, Nano Lett., 2012, 12, 414-419.

57 J. Maultzsch, H. Telg, S. Reich and C. Thomcen, Phys. Rev. B: Condens. Matter Mater. Phys., 2005, 72, 205438.

58 A. Jorio, M. A. Pimenta, A. G. Souza Filho, R. Saito, G. Gresselhaus and M. S. Dresselhaus, New J. Phys., 2003, 5, 139.

59 G. U. Sumanasekera, J. L. Allen, S. L. Fanag, A. L. Loper, A. M. Rao and P. C. Eklund, J. Phys. Chem. B, 1999, 103, 4292-4297.

60 T. Liu and S. Kumar, Chem. Phys. Lett., 2003, 378, 257-262. 61 C. L. Pint, Y. Q. Xu, E. Morosan and R. H. Hauge, Appl. Phys. Lett., 2009, 94(18), 182107.

62 B. Zhao, D. N. Futaba, S. Yasuda, M. Akoshima, T. Yamada and K. Hata, ACS Nano, 2009, 3(1), 108-114. 
63 D. N. Futaba, K. Hata, T. Yamada, T. Hiraoka, Y. Hayamizu, Y. Kakudate, O. Tanaike, H. Hatori, M. Yumura and S. Iijima, Nat. Mater., 2006, 5(12), 987-994.

64 C. T. Lin, C. Y. Lee, T. S. Chin, R. Xiang, K. Ishikawa, J. Shiomi and S. Maruyama, Carbon, 2011, 49, 1446-1452.

65 J. N. Tey, X. Ho and J. Wei, Nanoscale Res. Lett., 2012, 7, 548. 66 Y. Yosida and I. Oguro, J. Appl. Phys., 1999, 86, 999-1003.

67 T. Takano, T. Takenobu and Y. Iwasa, J. Phys. Soc. Jpn., 2008, 77, 124709-124713.

68 N. F. Mott, Metal-Insulator Transitions, Taylor and Francis, London, 1990, pp. 65-105.

$69 \mathrm{~J} . \mathrm{Wu}, \mathrm{H}$. Schmidt, K. K Amara, X. Xu, G. Eda and B. Ozyilmaz, Nano Lett., 2014, 14(5), 2730-2734.

70 M. Caban-Acevedo, D. Liang, K. S. Chew, J. P. DeGrave, N. S. Kaiser and S. Jin, ACS Nano, 2013, 7(2), 1731-1739.

71 J. Hone, Top. Appl. Phys., 2001, 80, 273-286.

72 L. X. Benedict, S. G. Louie and M. L. Cohen, Solid State Commun., 1996, 100(3), 177-180.

73 A. M. Marconnet, M. A. Panzer and K. E. Goodson, Rev. Mod. Phys., 2013, 85(3), 1296-1327.

74 X. Huang, J. Wang, G. Eres and X. Wang, Carbon, 2011, 49, 1680-1691.

75 S. Maruyama, Microscale Thermophys. Eng., 2003, 7, 41-50.

76 M. S. Dresselhaus, G. Dresselhaus, J. C. Charlier and E. Herneandez, Philos. Trans. R. Soc., A, 2004, 362, 2065-2098. 77 T. Tohei, A. Kuwabar, F. Oba and I. Tanaka, Phys. Rev. B: Condens. Matter Mater. Phys., 2006, 73, 064304.

78 J. M. Simmons, B. M. Nichols, S. E. Baker, M. S. Marcus, O. M. Castellini, C.-S. Lee, R. J. Hamers and M. A. Eriksson, J. Phys. Chem. B, 2006, 110, 7113-7118.
79 M. Grujicic, G. Cao and R. Singh, Appl. Surf. Sci., 2003, 211, 166-183.

80 J. W. Suk, W. H. Lee, J. Lee, H. Chou, R. D. Piner, Y. Hao, D. Akinwande and R. S. Ruoff, Nano Lett., 2013, 13(4), 1462-1467.

81 A. Pirkle, J. Chan, A. Venugopal, D. Hinojos, C. W. Magnuson, S. McDonnell, L. Colombo, E. M. Vogel, R. S. Ruoff and R. M. Wallace, Appl. Phys. Lett., 2011, 99, 122108.

82 R. D. Bernard, Thermoelectricity in Metal and Alloy, Taylor and Francis, London, 1992.

83 M. J. Burns and P. M. Chaikin, J. Phys. C: Solid State Phys., 1985, 18, 743-749.

84 A. B. Kaiser, Y. W. Park, G. T. Kim, E. S. Choi, G. Dusberg and S. Roth, Synth. Met., 1999, 103, 2547-2550.

85 J. Hone, I. Ellwood, M. Muno, A. Mizel, M. L. Cohen and A. Zettl, Phys. Rev. Lett., 1998, 80(5), 1042-1045.

86 O. L. Tirpak, W. V. Schoenfeld and L. Chernyak, Appl. Phys. Lett., 2006, 88, 202110.

87 A. Duzynska, A. Taube, K. P. Korona, J. Judek and M. Zdrojek, Appl. Phys. Lett., 2015, 106, 183108.

88 S. Sahoo, V. R. Chitturi, R. Agarwal, J. W. Jiang and R. S. Katiyar, ACS Appl. Mater. Interfaces, 2014, 6, 19958-19965.

89 J. Hone, M. Whitney, C. Piskoti and A. Zettl, Phys. Rev. B: Condens. Matter Mater. Phys., 1999, 59(4), 2514-2516.

90 F. Lian, J. P. Llinas, Z. Li, D. Estrada and E. Pop, Appl. Phys. Lett., 2016, 108, 103101.

91 M. T. Pettes, I. Jo, Z. Yao and L. Shi, Nano Lett., 2011, 11, 1195-1200.

92 F. D. Li, F. Y. Hui, C. Yang, L. Wei and Z. X. Xin, Chin. Phys. B, 2013, 22(1), 016501. 